\title{
Cell Death and Cell Proliferation in Cartilage Layers in Human Anterior Cruciate Ligament Tibial Insertions after Rupture
}

Hirotaka Mutsuzaki ${ }^{1}$, Masataka Sakane ${ }^{2}, \quad$ Katsuya Honda ${ }^{3}, \quad$ Kotaro Ikeda ${ }^{4}$, Shinya Hattori ${ }^{5}$, and Naoyuki Ochiai ${ }^{2}$

${ }^{1}$ Department of Orthopaedic Surgery, Ibaraki Prefectural University of Health Sciences, 4669-2 Ami Ami-machi, Inashiki-gun, Ibaraki, 300-0394, Japan

${ }^{2}$ Department of Orthopaedic Surgery, Institute of Clinical Medicine, Graduate School of Comprehensive Human Sciences, University of Tsukuba,

1-1-1 Tennodai, Tsukuba, Ibaraki 305-8575, Japan

${ }^{3}$ Department of Legal Medicine, Doctoral Program in Social and Environmental Medicine, Graduate School of Comprehensive Human Sciences, University of Tsukuba, 1-1-1 Tennodai, Tsukuba, Ibaraki 305-8575, Japan

${ }^{4}$ Department of Orthopaedic Surgery, Ichihara Hospital,

3681 Ozone, Tsukuba, Ibaraki 300-3295, Japan

${ }^{5}$ Biomaterial Center, National Institute for Materials Science, 1-1 Namiki, Tsukuba, Ibaraki 305-0044, Japan

Corresponding Author:

Masataka Sakane, M.D., Ph.D.

Tel: +81-29-853-3219; Fax: +81-29-853-3214,

E-mail address: sakane-m@md.tsukuba.ac.jp 


\section{Abstract}

The purpose of this study is to investigate cellular responses and histological changes of cartilaginous layers in human anterior cruciate ligament (ACL) tibial insertions after rupture compared with those in normal insertions. Sixteen tibial insertions of ruptured ACLs were obtained during primary ACL reconstructions. We also obtained sixteen normal ACL tibial insertions from cadavers. Terminal deoxynucleotidyl transferase-mediated deoxyuridine triphosphate-biotin nick end labeling (TUNEL) to detect apoptosis, proliferating cell nuclear antigen (PCNA) staining, and histological examination were performed. The percentage of TUNEL-positive chondrocytes in ruptured ACL insertions (30.2 $\pm 15.6 \%$ ) was higher than that in normal insertions $(9.6 \pm 5.8 \%)$. The percentage of PCNA-positive chondrocytes was significantly different between ruptured ACL insertions (19.9 $\pm 15.0 \%)$ and normal insertions $(12.3 \pm 7.3 \%)$. The average thickness of the cartilage layer, the glycosaminoglycan-stained area, and the number of chondrocytes per millimeter in ruptured ACL insertions were smaller than those in normal insertions. The decrease in the number of chondrocytes owing to an imbalance between cell death and cell proliferation in the ACL insertions after rupture, as compared with normal insertions, may lead to histological changes of the cartilage layer in the insertions. An in-depth understanding of injured ACL insertion may help elucidate the etiology of histological changes and the function and significance of the existence of the cartilage layer of insertion, and may help in developing optimal treatment protocols for ACL injuries if apoptosis and cell proliferation are controlled.

Key words: anterior cruciate ligament - insertion - rupture - apoptosis - cell proliferation 


\section{Introduction}

The attachment zones of ligaments and tendons to the bone are highly important and among the most complex of all biologic tissues. Within a short distance, soft tissues are transformed into hard tissues by a progression of tissue types. The tibial insertion of a normal anterior cruciate ligament (ACL) is classified as a direct insertion consisting of an interfacial ligamentous structure, an uncalcified cartilage layer, a calcified cartilage layer, and bone. The functions of attachment zones are not well understood. However, the insertion at the soft tissue / hard tissue interface is considered to be efficient in transmitting tensile force, because of its two cartilage layers $(1,2)$. Injuries to the ACL often occur at the ligament-to-bone insertion site. Because the ACL exhibits a poor healing potential (3), surgical intervention is often required. An understanding of the structural and chemical properties of the ACL-to-bone insertion site is necessary for the prevention and early management of ACL injury. However, clinically, the etiology of this lesion remains unknown.

We reported that histological degenerative changes of the cartilage layers in ruptured ACL tibial insertions progress with time due to chondrocytes apoptosis in the insertion (4). Moreover, we reported that 2 and 4 weeks after ACL resection in rabbits, the average percentages of TUNEL-positive chondrocytes were significantly higher than those in the sham-operated group, and histological changes occurred 6 weeks after the resection (5). Homeostasis is achieved by balance between cell death and proliferation in tissue. Cell death and regulation of cell proliferation in ruptured human ACL insertions compared with those in normal insertions have not been reported. The purpose of this study is to investigate cellular responses and histological changes of cartilaginous layers in human anterior cruciate ligament (ACL) tibial insertions after rupture compared with those in normal insertions. 


\section{Materials and Methods}

Sixteen tibial insertions of an ACL remnant were obtained during primary human ACL reconstructions performed from January 2006 to July 2008 (16 patients; 16 knees; male, 7; female, 9; mean age, $22.2 \pm 6.7$ years). The duration from injury to operation was set up to 200 days (mean, $93.4 \pm 51.8$ days) similar to our previous report (4). All the subjects gave their written informed consent to participate in this study. The study was approved by the local ethics committee. The arthroscopic inspections of ligament remnants at the time of surgery showed that no substantial ACL remained or there was no attachment of the ACL to the femur, and the ACL and/or synovium was attached to the femur, but no apparent tension was present, as determined by probing. All specimens were obtained using an 8- to 10-mm-core reamer (Arthrex, Fl, USA) during the drilling of the tibial bone tunnel (4). We also obtained sixteen normal ACL tibial insertions from cadavers (13 cadavers; 16 knees; male, 9; female, 4; mean age, $45.1 \pm 14.9$ years). The duration from death to autopsy was from 12 to 102 hours (mean: $43.5 \pm 22.0$ hours). We performed medicolegal autopsy of the cadavers of people who died in traffic accidents. These people did not have ACL injury and were healthy in life. The patients' data (age, gender, number of days after injury, side of injury, and postmortem duration) are summarized in Table 1.

All the specimens were fixed in $20 \%$ neutral buffered formalin, decalcified, and embedded in paraffin, then sliced sagittally into 5- $\mu$ m-thick sections parallel to the surface of the cut. Hematoxylin and eosin (H\&E) staining, safranin-O staining for the identification of glycosaminoglycans (GAG) in the cartilage layer, and Masson’s trichrome (MT) staining for the identification of collagenous fiber tissue in ACL tibial insertions were then performed. 
Using an in situ apoptosis detection kit (ApopTag ${ }^{\circledR}$ Plus Peroxidase In-Situ Apoptosis Detection kit \#S7101, CHEMICON ${ }^{\circledR}$ International, Inc., USA and Canada), we performed terminal deoxynucleotidyl transferase-mediated deoxyuridine triphosphate-biotin nick end labeling (TUNEL) to detect DNA fragmentation, which is the biochemical hallmark of apoptosis (6). Following the TUNEL protocol, the specimens were incubated in an equilibration buffer for 10 minutes at room temperature, and then incubated with terminal deoxynucleotidyl transferase (TdT) for 60 minutes in a humidified chamber. An anti-digoxigenin antibody conjugate was then applied to the specimens at room temperature for another 60 minutes in a humidified chamber. Proliferating cell nuclear antigen (PCNA) immunostaining was performed in accordance with the method described by Suzuki et al (7). Deparaffinised sections were rinsed in PBS for 5 minutes. For antigen retrieval, sections were incubated in $0.01 \mathrm{M}$ Tris-0.001M EDTA buffer and heated at $95-99{ }^{\circ} \mathrm{C}$ in water bath for 40 minutes. They were then immersed in 3\% hydrogen peroxidase $\left(\mathrm{H}_{2} \mathrm{O}_{2}\right)$ in methanol for 15 minutes to block endogenous peroxidase. After slides were rinsed in PBS for 5 minutes, they were preblocked with a solution of $10 \%$ normal rabbit serum at room temperature for 10 minutes and incubated at room temperature for 1 hour with a monoclonal antibody to PCNA (PC-10, Code No. M879, DAKO, Denmark). As the negative control, normal nonimmune mouse serum was used as the primary antibody.

The cut specimens were examined using a light microscope (BX-51, Olympus Optical Co., Ltd., Tokyo, Japan) equipped with a CCD camera system (DP50, Olympus). The number of chondrocytes, the number of TUNEL-positive chondrocytes, and the number of PCNA-positive chondrocytes of the cartilage layers (uncalcified and calcified cartilage layers) in the ACL insertions were determined. We then determined the percentages of 
TUNEL-positive chondrocytes and PCNA-positive chondrocytes. Cartilage layer and GAG-stained areas were determined using the Mac Scope Program installed in a Macintosh computer (Mitani Co., Japan). We defined a cartilage layer in an ACL tibial insertion as the tissue with round cells between the ligament (fiber tissue was identified by H\&E and MT stainings) and the bone (lamellar bone tissue was identified by H\&E staining), and we defined a GAG-stained area in the insertion as the area stained red by safranin-O. Then, the areas of the cartilage layer and GAG-stained areas were determined. Next, the obtained values were divided by the width of the ACL tibial insertion of each specimen to correct the values. We defined the corrected values of the cartilage layer, the GAG-stained area, and number of chondrocytes as the average thickness of the cartilage layer, the average thickness of the GAG-stained area, and the number of chondrocytes per millimeter, respectively. We compared the percentage of TUNEL-positive chondrocytes, the percentage of PCNA-positive chondrocytes, the average thickness of the cartilage layer, the average thickness of the GAG-stained area, and the number of chondrocytes per millimeter between the ruptured ACL group and the normal ACL group. Our histomorphometrical analysis method was described in our previous report $(4,5)$. The data of the ruptured ACL group and the normal ACL group were compared using unpaired Student's $t$ test at a $p<0.05$ significance value.

\section{Results}

The histological sections of the ruptured ACL tibial insertion 81 days after the injury of a 20-year-old female were examined. A thin cartilage layer was observed (Fig. 1a). Small GAG-stained area was observed (Fig. 1b). Ligament fibers are thin and randomly distributed (Fig. 1c). Many TUNEL-positive chondrocytes were observed (Fig. 1d, e) in the 
cartilage layer of insertion. Many PCNA-positive chondrocytes were also observed in the cartilage layer (Figs. 1f, g).

The histological sections of the normal ACL tibial insertion of the right knee of a 35-year-old male were examined. Under the ligament layer, thick cartilage layers with numerous chondrocytes (Fig. 2a) and a thick GAG-stained area were observed (Fig. 2b). Thick ligament fibers with a regular linear arrangement were observed (Fig. 2c). Few TUNEL-positive chondrocytes were observed (Fig. 2d, e). PCNA-positive chondrocytes were observed in the cartilage layer (Figs. 2f, g).

The percentage of TUNEL-positive chondrocytes in the ruptured ACL group (30.2 $\pm 15.6 \%)$ was significantly higher than that in the normal ACL group $(9.6 \pm 5.8 \%)(p<$ 0.0001). The percentage of PCNA-positive chondrocytes was significantly different between the ruptured ACL group $(19.9 \pm 15.0 \%)$ and the normal ACL group $(12.3 \pm 7.3 \%)(p=$ 0.0393). The average thickness of the cartilage layer in the ruptured ACL group (152.3 \pm $146.5 \mu \mathrm{m})$ was smaller than that in the normal ACL group $(408.5 \pm 297.8 \mu \mathrm{m})(p=0.0022)$. The average thickness of the GAG-stained area in the ruptured ACL group (55.8 $\pm 76.7 \mu \mathrm{m})$ was also smaller than that in the normal ACL group $(172.8 \pm 191.2 \mu \mathrm{m})(p=0.0152)$. The number of chondrocytes per millimeter in the ruptured ACL group (25.3 \pm 16.4 ) was significantly smaller than that in the normal ACL group $(41.2 \pm 13.4)(p=0.0027)$. The histomorphometrical data of the tissues are summarized in Table 2. 


\section{Discussion}

The main role of apoptosis is to maintain homeostasis (8). Apoptosis is an important cellular event specifically controlled by a series of complex cascades $(9,10)$, in which various stimuli mediated by signals through many pathways induce cell death. Apoptosis, or programmed cell death, is a normal physiological process in healthy mature tissues in which the percentages of apoptotic cells range from 3\% to $10 \%$ (11). Previously, we reported that histological degenerative changes of the cartilage layer due to chondrocyte apoptosis in a ruptured ACL tibial insertion progress with time, particularly in the first 2 months (4). We also reported that 2 and 4 weeks after ACL resection in rabbits, the percentages of TUNEL-positive chondrocytes were significantly higher than those in the shamoperated group, and histological changes occurred 6 weeks after the resection (5). In our present study, we observed that approximately $30 \%$ of chondrocytes were apoptotic in ruptured ACL insertions and approximately $10 \%$ of chondrocytes in normal insertions. Osteocytes and chondrocytes undergo apoptosis $10-70 \mathrm{hr}$ after death in humans (12). In this study, the chondrocyte apoptosis rate in normal insertions was similar to those in previous reports of calcified cartilage layer in ACL insertion of rabbits (5) and articular cartilage of mice and rats (11). The chondrocyte apoptosis rate in normal insertions was lower than that in the ruptured ACL group. We considered that the possible factor inducing apoptosis in the cartilage layer of ACL tibial insertions is proteinases and/or stress deprivation rather than the effect of death in humans. Proteoglycan degradation and matrix metalloproteinase expression in a rabbit articular cartilage were shown to be associated with chondrocyte apoptosis in osteoarthritis (13). In an ACL-deficient knee, proteinases may be expressed because of the progression of osteoarthritis (14). However, cyclic tensile stress and compressive strain were shown to be 
associated with the apoptosis of fibroblasts, chondrocytes, and osteocytes $(6,15-18)$. The ACL tibial insertion after ACL rupture was considered to be a model of stress deprivation.

PCNA expression level is an index of cell number increase and PCNA is expressed from the late G1 phase to the S phase of the cell cycle (19). The percentage of PCNA-positive chondrocytes was significantly different between the ruptured ACL insertions and the normal insertions. The increase in the number of PCNA-positive cells in the ruptured ACL group was observed compared with the normal ACL group. We considered that the large average number of the PCNA-positive cells may be due to the response of the cells to the presence of a large average number of TUNEL-positive cells and/or active inflammation in knee instability.

Homeostasis is achieved by the balance between cell death and proliferation. In our study, because the normal ACL insertions had chondrocytes TUNELpositive (10\%) and chondrocytes PCNA-positive (12\%), 10\% of chondrocytes can turnover in the cartilage layers of the normal ACL insertions. Both cell death and cell proliferation may participate in maintaining homeostasis and structures in normal ACL insertion. However, TUNEL-positive chondrocytes (30\%) and PCNA-positive chondrocytes (20\%) were observed in the cartilage layer in the ACL ruptured group. Therefore, an 10\% decrease in the number of chondrocytes may occur in the cartilage layers in the ACL ruptured group. As a result, the average thickness of the cartilage layer and the average thickness of the GAG-stained area produced by the chondrocytes decreased in the ruptured group. These results suggest that the percentages of proliferating cells and apoptotic cells are closely related to the maintenance of the insertion structure in the normal group and degeneration of the insertion structure in the ruptured ACL group. The decrease in the number of chondrocytes from an imbalance between 
cell death and cell proliferation in the ACL insertions after ACL rupture compared with the normal insertionsmay lead to histological degenerative changes of the cartilage layer in the insertions.

The amount of matrix and the number of chondrocytes differ between mature and immature bovine (20). In this study, we used the specimens after the growth plate is closed. In this study the growth plates of all specimens in the two groups were closed. In other words, all specimens in the two groups can be considered as mature. We considered that the amounts of matrix and number of chondrocytes were unrelated to age after the growth plate was closed. Therefore, we compared these two groups, even while noting a higher age in the control group than in the ACL ruptured group. We observed statistically significant differences in cartilage layer thickness, GAG-stained area thickness, chondrocyte number, and chondrocyte apoptosis rate between the ruptured ACL group and the control. Therefore, the ACL insertion can change owing to ACL rupture rather than aging.

An in-depth understanding of injured ACL insertion may help elucidate the etiology of histological changes and the function and significance of the existence of the cartilage layer of insertion. This knowledge may help in developing optimal treatment protocols for ACL injuries if apoptosis and cell proliferation are controlled.

In the future, the interactions among chemical reactions and mechanical stimulation after ligament injury should be investigated. Analysis in much earlier phases after an injury is necessary. It is unclear whether apoptosis in ruptured ACL insertions affects the surrounding articular cartilage. Furthermore, changes and apoptosis in the surrounding articular cartilage should be investigated. 


\section{Acknowledgements}

The authors thank the Core Research for Evolutional Science and Technology (CREST) Program, and the Leading Project (LP) of the Ministry of Education, Culture, Sports, Science and Technology of Japan for financial support. 


\section{References}

(1) Benjamin, M., Evans, E.J., and Copp L. (1986) The Histology of Tendon Attachments to Bone in Man J. Anat. 149:89-100.

(2) Cooper, R.R., and Misol, S. (1970) Tendon and Ligament Insertion: a Light and Electron Microscopic Study J. Bone Joint Surg. Am. 52:1-20.

(3) Murray, M.M., Martin, S.D., Martin, T.L., and Spector, M. (2000) Histological Changes in the Human Anterior Cruciate Ligament after Rupture J. Bone Joint Surg. Am. 82:1387-1397.

(4) Mutsuzaki, H., Sakane, M., Ikeda, K., Ishii, T., Hattori, S., Tanaka, J., and Ochiai, N. (2007) Histological Changes and Apoptosis of Cartilage Layer in Human Anterior Cruciate Ligament Tibial Insertion after Rupture Knee Surg. Sports Traumatol. Arthrosc. 15:602-609.

(5) Hattori, S., Sakane, M., Mutsuzaki, H., Tanaka, J., Ochiai, N., and Nakajima, H. (2007) Chondrocyte Apoptosis and Decrease of Glycosaminoglycan in Cranial Cruciate Ligament Insertion after Resection in Rabbits J. Veter. Med. Sci. 69:253-258.

(6) Tuoheti, Y., Itoi, E., Pradhan, R.L., Wakabayashi, I., Takahashi, S., Minagawa, H., Kobayashi, M., Okada, K., and Shimada, Y. (2005) Apoptosis in the Supraspinatus Tendon with Stage II Subacromial Impingement J. Shoulder Elbow Surg. 14:535-541.

(7) Suzuki, K., Katoh, R., and Kawaoi, A. (1992) Immunohistochemical Demonstration of Proliferating Cell Nuclear Antigen (PCNA) in Formalin-Fixed, Paraffin-Embedded Sections from Rat and Human Tissues Acta Histochem. Cytochem. 25:13-21.

(8) Cohen, J.J. (1993) Apoptosis Immunol. Today 14:126-130.

(9) Golstein, P. (1997) Controlling Cell Death Science 275:1081-1082.

(10) Green, D.R., and Reed, J.C. (1998) Mitochondria and Apoptosis Science 281:1309-1312.

(11) Adams, C.S., and Horton, W.E.Jr. (1998) Chondrocyte Apoptosis Increases with Time 
Age in the Articular Cartilage of Adult Animals Anat. Rec. 250:418-425.

(12) Wakasugi, C. (2005) Legal Medicine, 4 th ed pp.333 Kyoto: Kinpoudou Press.

(13) Sabatini, M., Rolland, G., Léonce, S., Thomas, M., Lesur, C., Pérez, V., de Nanteuil, G., and Bonnet J. (2000) Effects of Ceramide on Apoptosis, Proteoglycan Degradation, and Matrix Metalloproteinase Expression in Rabbit Articular Cartilage Biochem. Biophys. Res. Commun. 267:438-444.

(14) Spindler, K.P., Clark, S.W., Nanney, L.B., and Davidson, J.M. (1996) Expression of Collagen and Matrix Metalloproteinases in Ruptured Human Anterior Cruciate Ligament: an in situ Hybridization Study J. Orthop. Res. 14:857-861.

(15) Ariga, K., Yonenobu, K., Nakase, T., Hosono, N., Okuda, S., Meng, W., Tamura, Y., and Yoshikawa, H. (2003) Mechanical Stress-Induced Apoptosis of Endplate Chondrocytes in Organ-Cultured Mouse Intervertebral Discs: an ex vivo Study Spine 28:1528-1533.

(16) Arnoczky, S.P., Tian, T., Lavagnino, M., Gardner, K., Schuler, P., and Morse, P. (2002) Activation of Stress-Activated Protein Kinases (SAPK) in Tendon Cells Following Cyclic Strain: the Effects of Strain Frequency, Strain Magnitude, and Cytosolic Calcium J. Orthop. Res. 20:947-952.

(17) Noble, B.S., Peet, N., Stevens, H.Y., Brabbs, A., Mosley, J.R., Reilly, G.C., Reeve, J., Skerry, T.M., and Lauyon, L.E. (2003) Mechanical Loading: Biphasic Osteocyte Survival and Targeting of Osteoclasts for Bone Destruction in Rat Cortical Bone Am. J. Physiol. Cell. Physiol. 284:C934-943.

(18) Soslowsky, L.J., Thomopoulos, S., Tun, S., Flanagan, C.L., Keefer, C.C., Mastaw, J., and Carpenter, J.E. (2000) Overuse Activity Injuries the Supraspinatus Tendon in an Animal Model: a Histologic and Biomechanical Study J. Shoulder Elbow Surg. 9:79-84. 
(19) Hall, P.A., Levison, D.A., Woods, A.L., Yu, C.C., Kellock, D.B., Watkins, J.A., Barnes, D.M., Gillett, C.E., Camplejohn, R., and Dover, R. (1990) Proliferating Cell Nuclear Antigen (PCNA) Immunolocalization in Paraffin Sections: an Index of Cell Proliferation with Evidence of Deregulated Expression in Some Neoplasma J. Pathol. 162:285-294.

(20) Wang, I.N., Mitroo, S., Chen, F.H., Lu, H.H., and Doty, S.B. (2006) Age-Dependent Changes in Matrix Composition and Organization at the Ligament-to-Bone Insertion $J$. Orthop. Res. 24:1745-1755. 


\section{Figure legends}

Figure 1.

Histological sections of ACL tibial insertion 81 days after injury from typical specimen.

a) H\&E staining, b) Safranin-O staining, c) MT staining, d)e) TUNEL, f)g) PCNA staining.

A thin cartilage layer is observed (Fig. 1a). Small GAG-stained area was observed (Fig. 1b). Ligament fibers are thin and randomly distributed (Fig. 1c). Many TUNEL-positive chondrocytes (arrows) are observed in the insertion (49.5\%) (Fig. 1d, e). PCNA-positive chondrocytes (arrows) are observed in the cartilage layer (28.5\%) (Fig. 1f, g). Fig. 1e is a magnification of the boxed part in Fig. 1d. Fig. 1g is a magnification of the boxed part in Fig. 1f.

Figure 2.

Histological sections of normal ACL tibial insertion from typical specimen.

a) H\&E staining, b) Safranin-O staining, c) MT staining, d)e) TUNEL, f)g) PCNA staining. Under the ligament layer, thick cartilage layers with numerous chondrocytes (Fig. 2a) and a thick GAG-stained area are observed (Fig. 2b). Thick ligament fibers with a regular linear arrangement are observed (Fig. 2c). Few TUNEL-positive chondrocytes (arrows) are observed (11.8\%) (Fig. 2d, e). PCNA-positive chondrocytes (arrows) are observed in the cartilage layer (15.9\%) (Fig. 2f, g). Fig. 2e is a magnification of the boxed part in Fig. 2d. Fig. $2 \mathrm{~g}$ is a magnification of the boxed part in Fig. $2 \mathrm{f}$. 


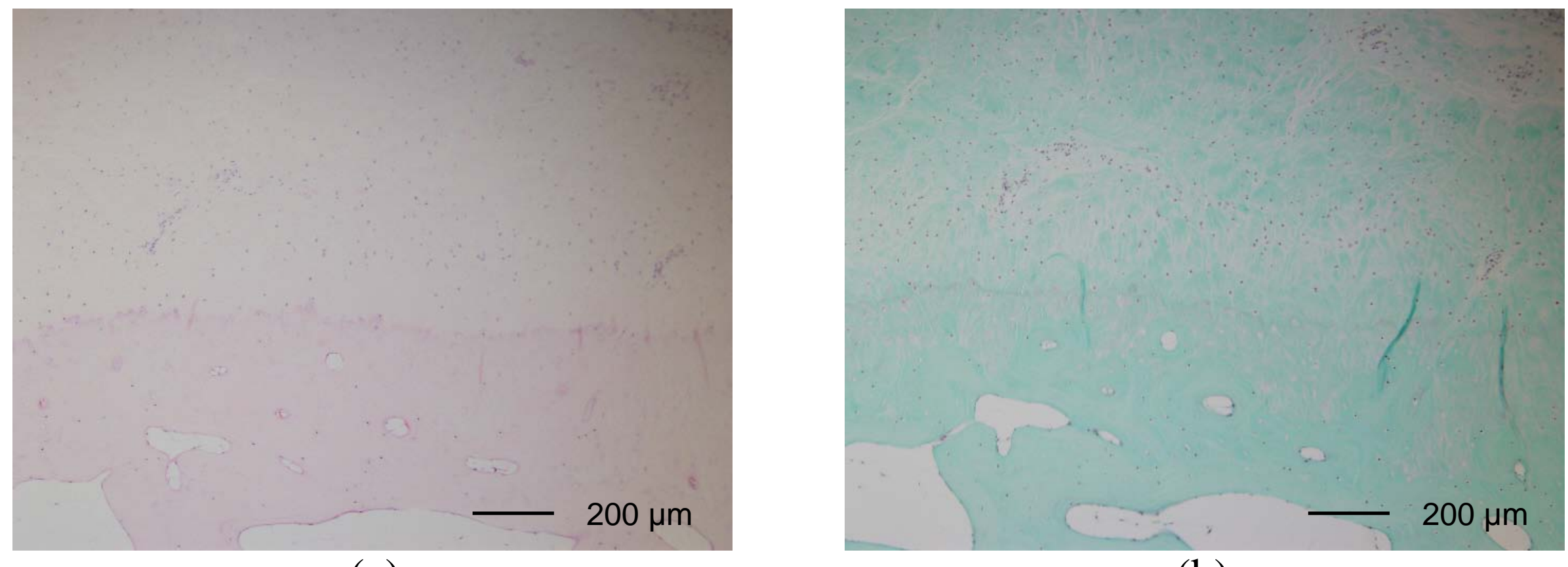

(a)

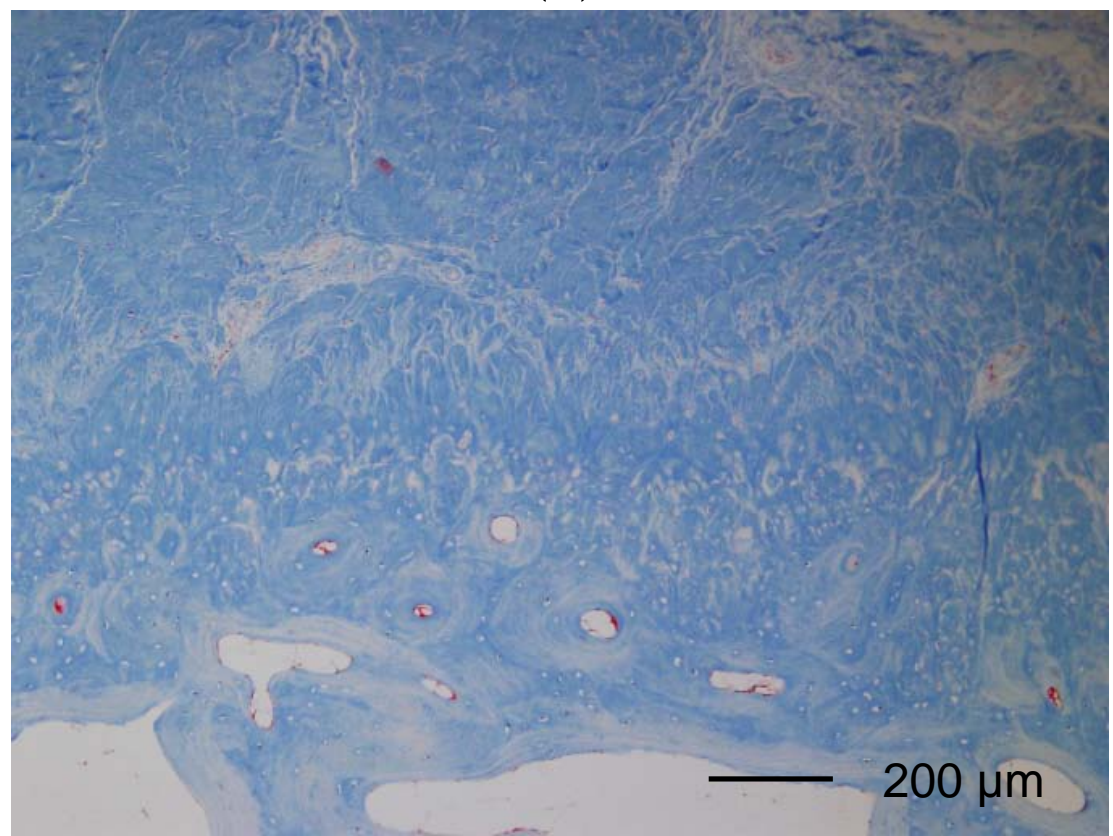

(b)

(c)

Fig. 1 


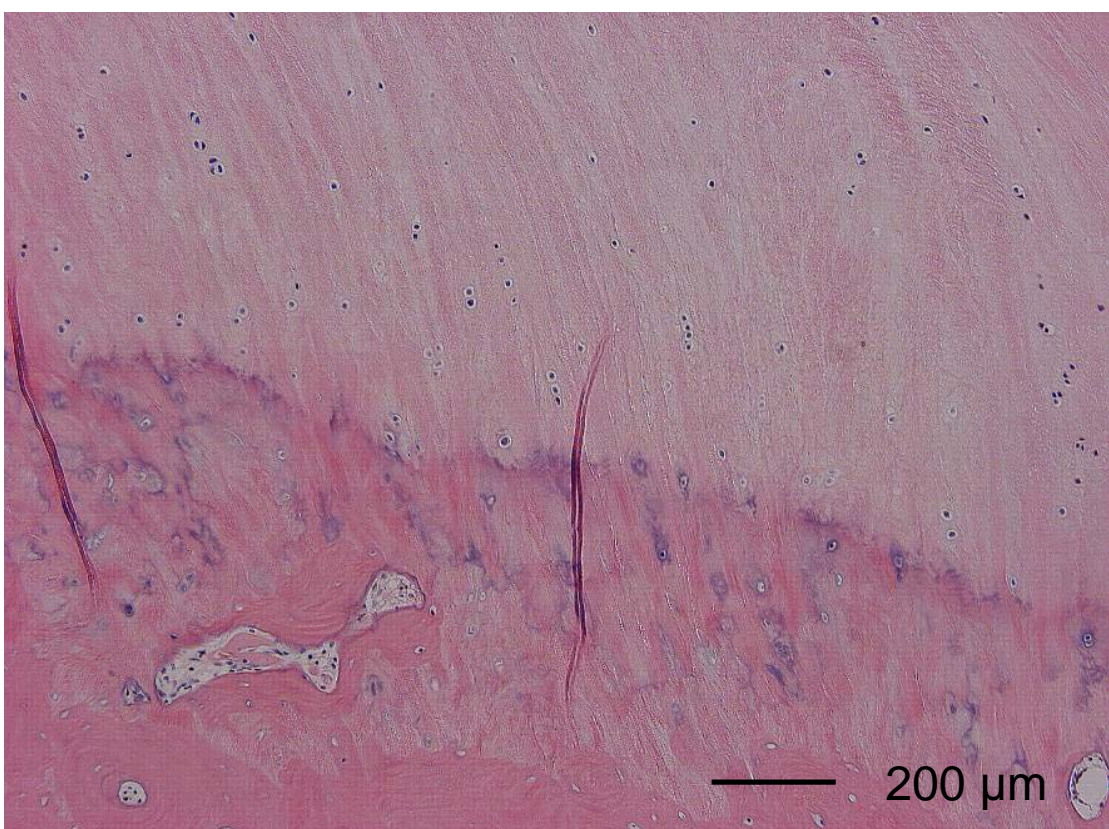

(a)

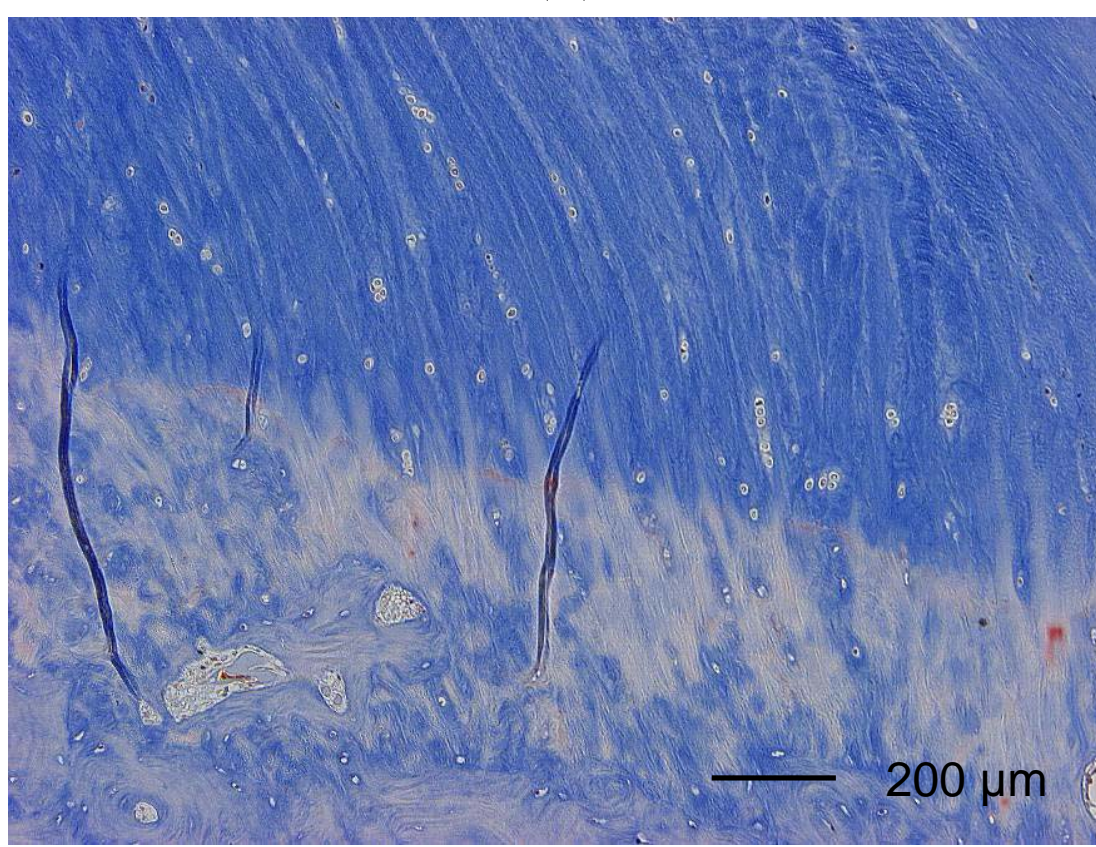

(c)
Fig. 2 


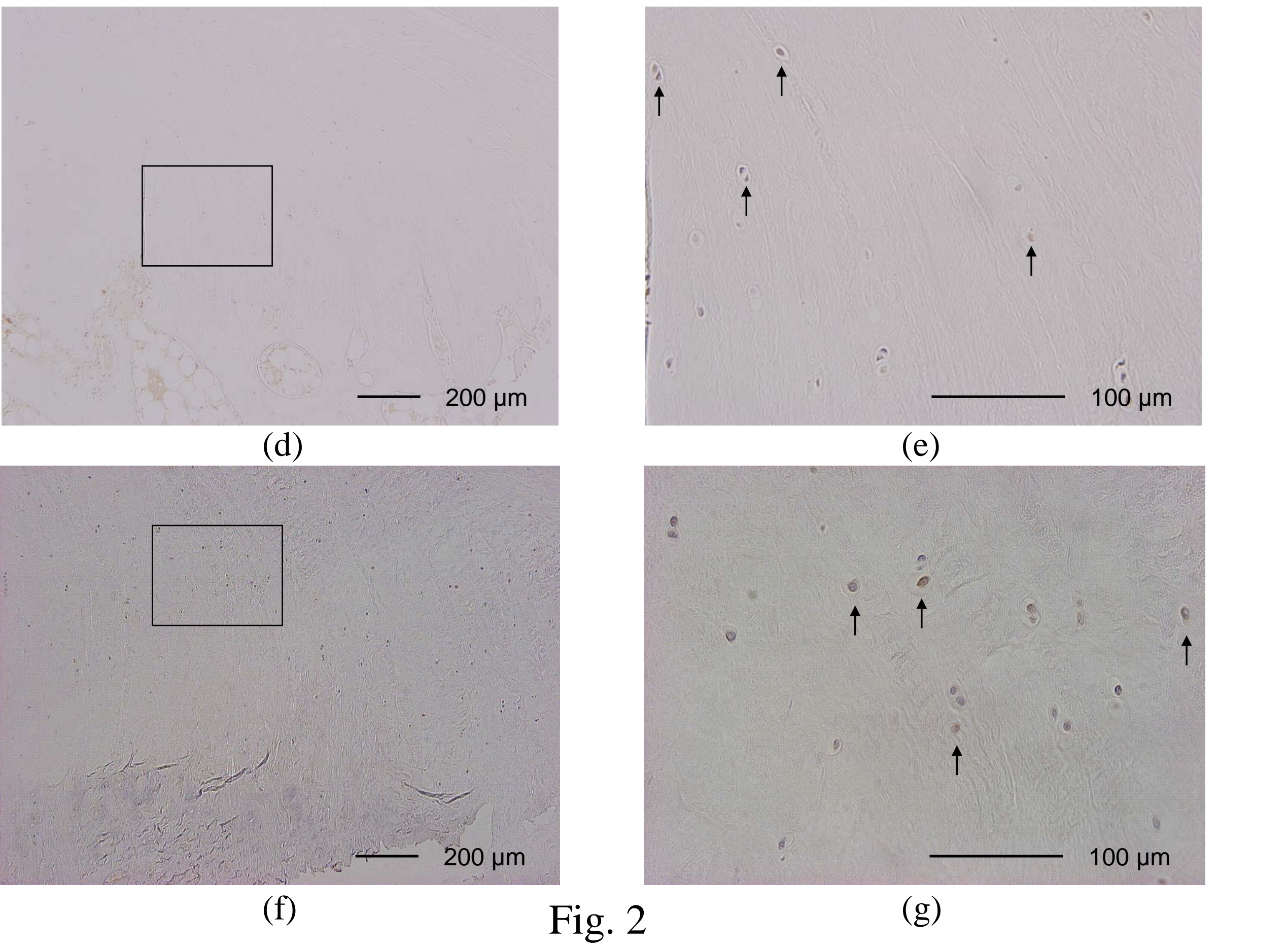


Table 1. Patients' data

\begin{tabular}{|c|c|c|c|c|c|c|c|c|c|}
\hline \multicolumn{4}{|c|}{ Ruptured ACL group } & \multicolumn{6}{|c|}{ Normal ACL group } \\
\hline Knee No.Patient No & Age & Gender & $\begin{array}{c}\text { Number of } \\
\text { days after } \\
\text { injury }\end{array}$ & Knee No & tient No & Age & Gender & Side & $\begin{array}{c}\text { postmortem } \\
\text { duration } \\
\text { (hours) }\end{array}$ \\
\hline 1 & 27 & M & 67 & 1 & 1 & 42 & M & $\mathrm{R}$ & 50 \\
\hline 2 & 20 & M & 71 & 2 & 2 & 54 & M & $\mathrm{R}$ & 28 \\
\hline 3 & 17 & M & 72 & 3 & 3 & 30 & $\mathrm{~F}$ & $\mathrm{R}$ & 28 \\
\hline 4 & 22 & $\mathrm{~F}$ & 82 & 4 & 4 & 61 & M & $\mathrm{R}$ & 48 \\
\hline 5 & 35 & M & 90 & 5 & 5 & 65 & M & $\mathrm{R}$ & 102 \\
\hline 6 & 19 & M & 97 & 6 & 6 & 52 & M & $\mathrm{R}$ & 30 \\
\hline 7 & 26 & $\mathrm{~F}$ & 103 & 7 & 7 & 63 & $\mathrm{~F}$ & $\mathrm{R}$ & 12 \\
\hline 8 & 17 & $\mathrm{M}$ & 104 & 8 & 8 & 26 & $\mathrm{~F}$ & $\mathrm{~L}$ & 48 \\
\hline 9 & 14 & $\mathrm{~F}$ & 122 & 9 & 9 & 32 & $\mathrm{~F}$ & $\mathrm{R}$ & 30 \\
\hline 10 & 19 & M & 128 & 10 & 10 & 35 & $\mathrm{M}$ & $\mathrm{R}$ & 30 \\
\hline 11 & 26 & M & 137 & 11 & & & & $\mathrm{~L}$ & \\
\hline 12 & 19 & M & 144 & 12 & 11 & 63 & M & $\mathrm{R}$ & 50 \\
\hline 13 & 25 & $\mathrm{M}$ & 179 & 13 & 12 & 30 & $\mathrm{M}$ & $\mathrm{R}$ & 55 \\
\hline 14 & 18 & $\mathrm{M}$ & 179 & 14 & & & & $\mathrm{~L}$ & \\
\hline 15 & 26 & $\mathrm{~F}$ & 199 & 15 & 13 & 33 & $\mathrm{M}$ & $\mathrm{R}$ & 54 \\
\hline 16 & 16 & $\mathrm{M}$ & 206 & 16 & & & & $\mathrm{~L}$ & \\
\hline Ave. & 21.6 & & 123.8 & Ave. & & 42.3 & & & 43.5 \\
\hline S.D. & 5.5 & & 46.4 & S.D. & & 14.3 & & & 22.0 \\
\hline
\end{tabular}


Table 2. The histomorphometrical data

\begin{tabular}{cccc}
\hline & Ruptured ACL group & Normal ACL group & $* p$ value \\
\hline \hline TUNEL-positive chondrocytes $(\%)$ & $43.6 \pm 14.8$ & $9.6 \pm 5.8$ & $p<0.00001$ \\
PCNA-positive chondrocytes $(\%)$ & $23.1 \pm 26.3$ & $12.3 \pm 7.3$ & $p=0.12383$ \\
average thickness of cartilage layer $(\mu \mathrm{m})$ & $175.2 \pm 142.7$ & $408.5 \pm 297.8$ & $p=0.00830$ \\
average thickness of GAG-stained area $(\mu \mathrm{m})$ & $43.2 \pm 66.8$ & $172.8 \pm 191.2$ & $p=0.01575$ \\
number of chondrocytes per millimeter & $21.5 \pm 12.1$ & $41.2 \pm 13.4$ & $p=0.00014$ \\
\end{tabular}

* Unpaired Student's t test 